\title{
LOWER BOUNDS FOR THE FIRST EIGENVALUE OF THE LAPLACIAN ON KÄHLER MANIFOLDS
}

\author{
XIAOLONG LI AND KUI WANG
}

\begin{abstract}
We establish lower bound for the first nonzero eigenvalue of the Laplacian on a closed Kähler manifold in terms of dimension, diameter, and lower bounds of holomorphic sectional curvature and orthogonal Ricci curvature. On compact Kähler manifolds with boundary, we prove lower bounds for the first nonzero Neumann or Dirichlet eigenvalue in terms of geometric data. Our results are Kähler analogues of well-known results for Riemannian manifolds.
\end{abstract}

Dedicated to Professor Richard Schoen on the occasion of his 70th birthday.

\section{INTRODUCTION}

Let $\left(M^{n}, g\right)$ be an $n$-dimensional compact connected Riemannian manifold, possibly with a smooth nonempty boundary $\partial M$. Denote by $\Delta$ the Laplacian of the metric $g$. In this paper, we shall consider the following three eigenvalue problems on the manifold $(M, g)$. When $\partial M=\emptyset$, the closed eigenvalue problem seeks all real numbers $\lambda$ for which there are nontrivial solutions $u \in C^{2}(M)$ to the equation

$$
\Delta u=-\lambda u \text {. }
$$

When $\partial M \neq \emptyset$, the Dirichlet eigenvalue problem is to find all real numbers $\lambda$ for which there are nontrivial solutions $u \in C^{2}(M) \cap C^{0}(\bar{M})$ to (1.1), subject to the boundary condition

$$
u=0 \text { on } \partial M
$$

and the Neumann eigenvalue problem looks for all real numbers $\lambda$ for which there are nontrivial solutions $u \in C^{2}(M) \cap C^{1}(\bar{M})$ to (1.1), subject to the boundary condition

$$
\frac{\partial u}{\partial \nu}=0 \text { on } \partial M
$$

where $\nu$ denotes the outward unit normal vector fields of $\partial M$. One can also consider these eigenvalue problems on a compact Kähler manifold by viewing it as a Riemannian manifold.

It is well-known that the spectrum of the Laplacian on a closed manifold consists of pure point spectrum $\left\{\mu_{i}\right\}_{i=0}^{\infty}$ that can be arranged in the order

$$
0=\mu_{0}<\mu_{1} \leq \mu_{2} \leq \mu_{3} \leq \cdots \rightarrow \infty .
$$

2010 Mathematics Subject Classification. 35P15, 53C55.

Key words and phrases. Eigenvalue comparison, modulus of continuity, orthogonal Ricci curvature.

The research of the second author is supported by NSFC No.11601359. 
The same holds for the spectrum with Neumann boundary condition. The Dirichlet spectrum consists of pure point spectrum $\left\{\lambda_{i}\right\}_{i=0}^{\infty}$ that can be arranged in the order

$$
0<\lambda_{1}<\lambda_{2} \leq \lambda_{3} \leq \cdots \rightarrow \infty .
$$

The study of the first nonzero eigenvalues is an important issue in both mathematics and physics, since this constant determines the convergence rate of numerical schemes in numerical analysis, describes the energy of a particle in the ground state in quantum mechanics, and determines the decay rate of certain heat flows in thermodynamics. Given its physical and mathematical significance, numerous bounds have been established for the first nonzero eigenvalue of the Laplacian on a compact Riemannian manifold (with Dirichlet or Neumann boundary conditions if the boundary is nonempty). We refer the reader to the books Cha84 SY94 for classical results and the surveys [LL10] And15, for later developments.

However, there are very few results for Kähler manifolds unless they are viewed as Riemannian manifolds. Lichnerowicz [Lic58] showed that if $M$ is closed Kähler manifold with Ric $\geq(n-1) \kappa>0$, then $\mu_{1} \geq 2(n-1) \kappa$. Note that this is a remarkable improvement of his well-known result in the Riemannian case, which asserts that $\mu_{1} \geq n \kappa$ if $M$ is a closed Riemannian manifold with Ric $\geq(n-1) \kappa>0$. Both his results are sharp as they are achieved by the complex projective space with the Fubini-Study metric and the sphere with the round metric, respectively. The proofs are simple applications of the Bochner formula or its Kähler version Lic58 (see also [Li12, Theorem 5.1] for the Riemannian case and Bal06, Theorem 6.14] for the Kähler case, and also Ura87 for an alternative proof via harmonic maps in the Kähler case). Moreover, both Lichnerowicz's results hold for the first Dirichlet eigenvalue of the Laplacian if the boundary is convex. The Riemannian one is proved by Reilly [Rei77] and the Kähler one is a recent result of Guedj, Kolev and Yeganefar GKY13. Finally, we would like to mentioned that these results for Kähler manifolds have been generalized to the $p$-Laplacian by Blacker and Seto BS19 by establishing a $p$-Reilly formula. More precisely, they proved that if $M$ is a closed Kähler manifold (or with a convex boundary) with Ric $\geq \kappa>0$, then the first nonzero eigenvalue (or first Dirichlet eigenvalue) of the $p$-Laplacian is bounded from below by $\left(\frac{p+2}{p(p-1)}(n-1) \kappa\right)^{\frac{p}{2}}$ provided that $p \geq 2$.

The above-mentioned results indicate that if one takes the Kählerity into consideration, then the lower bound should be improved, as least in the positive curvature case. In general, lower bounds that are sharp for Riemannian manifolds are not sharp for Kähler manifolds. Therefore, it is the purpose of this article to investigate the first nonzero eigenvalue of the Laplacian on a compact Kähler manifold and establish lower bounds that reflect the Kähler structure.

Lichnerowicz's Riemannian result is a special case of the following optimal lower bound on the first nonzero eigenvalue of the Laplacian in terms of dimension, diameter, and Ricci curvature lower bound.

Theorem 1.1. Let $\left(M^{n}, g\right)$ be a compact Riemannian manifold (possibly with a smooth convex boundary) with diameter $D$ and Ric $\geq(n-1) \kappa$ for $\kappa \in \mathbb{R}$. Let $\mu_{1}$ be the first nonzero eigenvalue of the Laplacian on $M$ (with Neumann boundary condition if $\partial M \neq \emptyset)$. Then

$$
\mu_{1} \geq \bar{\mu}_{1}(n, \kappa, D)
$$


where $\bar{\mu}_{1}(n, k, D)$ is the first nonzero Neumann eigenvalue of the one-dimensional eigenvalue problem:

$$
\varphi^{\prime \prime}-(n-1) T_{\kappa} \varphi^{\prime}=-\lambda \varphi
$$

on the interval $[-D / 2, D / 2]$.

Here and in the rest of this article, the function $T_{\kappa}$ is defined for $\kappa \in \mathbb{R}$ by

$$
T_{\kappa}(t)= \begin{cases}\sqrt{\kappa} \tan (\sqrt{\kappa} t), & \kappa>0, \\ 0, & \kappa=0, \\ -\sqrt{-\kappa} \tanh (\sqrt{-\kappa} t), & \kappa<0 .\end{cases}
$$

The above theorem is proved by Zhong and Yang for the $\kappa=0$ case and by Kröger Krö92 for general $\kappa \in \mathbb{R}$ (see also Bakry and Qian BQ00 for the above explicit form and extensions to smooth metric measure spaces). These works use the gradient estimates method initiated by Li Li79] and Li and Yau LY80. In 2013, Andrews and Clutterbuck AC13 gave a simple proof via the modulus of continuity estimates (see also [WW17 for an elliptic proof based on [AC13 and Ni13).

The first main theorem of this paper is the following Kähler analogue of the above theorem.

Theorem 1.2. Let $\left(M^{m}, g, J\right)$ be a compact Kähler manifold of complex dimension $m$ and diameter $D$ whose holomorphic sectional curvature is bounded from below by $4 \kappa_{1}$ and orthogonal Ricci curvature is bounded from below by $2(m-1) \kappa_{2}$ for some $\kappa_{1}, \kappa_{2} \in \mathbb{R}$. Let $\mu_{1}$ be the first nonzero eigenvalue of the Laplacian on $M$ (with Neumann boundary condition if $M$ has a strictly convex boundary). Then

$$
\mu_{1} \geq \bar{\mu}_{1}\left(m, \kappa_{1}, \kappa_{2}, D\right),
$$

where $\bar{\mu}_{1}\left(m, \kappa_{1}, \kappa_{2}, D\right)$ is the first Neumann eigenvalue of the one-dimensional eigenvalue problem

$$
\varphi^{\prime \prime}-\left(2(m-1) T_{\kappa_{2}}+T_{4 \kappa_{1}}\right) \varphi^{\prime}=-\lambda \varphi
$$

on $[-D / 2, D / 2]$.

Instead of lower bounds of Ricci curvature, we use lower bounds of holomorphic sectional curvature and orthogonal Ricci curvature (see Section 2 for definitions) in Theorem 1.2. These curvature conditions are more suitable for Kähler manifolds as they reflect more on the Kähler structure, particularly for various comparison theorems. For example, the Laplacian comparison theorem in Riemannian geometry assumes only Ricci lower bounds and are sharp on space forms of constant sectional curvature, while the Laplacian comparison theorem on Kähler manifolds that are sharp on complex space forms of constant holomorphic sectional curvature requires lower bounds of both holomorphic sectional curvature and orthogonal Ricci curvature. The latter was established until recently by Ni and Zheng in NZ18, and was known previously under the stronger condition of bisectional curvature lower bounds by the work of Li and Wang [LW05] (see also [TY12] for Hessian comparison theorem under bisectional curvature lower bounds). Since both Theorem 1.1 and 1.2 can be viewed as eigenvalue comparison theorems, it is natural to work with lower bounds of holomorphic sectional curvature and orthogonal Ricci curvature. 
Our proof of Theorem 1.2 uses the modulus of continuity approach introduced by Andrews and Clutterbuck in [AC13. Lower bounds of the first nonzero eigenvalue are derived as large time implication of the modulus of continuity estimates, which in turn relies on a comparison theorem for the second derivatives of $d(x, y)$ as a function on $M \times M$.

To the best of our knowledge, Theorem 1.2 is the first lower bound for the first nonzero eigenvalue of the Laplacian on Kähler manifolds that depends on the diameter. It also seems to be the first lower bound for the Neumann boundary condition in the Kähler setting. Moreover, the monotonicty of $\bar{\mu}_{1}\left(m, \kappa_{1}, \kappa_{2}, D\right)$ in $D$ implies that when the diameter is small, the lower bound provided in Theorem 1.2 is better than any lower bound that is independent of the diameter.

Expressing $\bar{\mu}_{1}\left(m, \kappa_{1}, \kappa_{2}, D\right)$ in terms of elementary functions does not seem to be possible, so we provide some explicit bounds and estimates in the next proposition for the purpose of comparing with other results.

Proposition 1.1. Let $\bar{\mu}_{1}\left(m, \kappa_{1}, \kappa_{2}, D\right)$ be as in Theorem 1.2. Then we have

$$
\bar{\mu}_{1}\left(m, \kappa_{1}, \kappa_{2}, D\right) \begin{cases}=\frac{\pi^{2}}{D^{2}}, & \text { if } \kappa_{1}=\kappa_{2}=0 ; \\ \geq \bar{\mu}_{1}\left(m, \kappa_{1}, 0, \frac{\pi}{2 \sqrt{\kappa_{1}}}\right)=8 \kappa_{1}, & \text { if } \kappa_{1}>0, \kappa_{2}=0 ; \\ \geq \bar{\mu}_{1}\left(m, 0, \kappa_{2}, \frac{\pi}{\sqrt{\kappa_{1}}}\right)=(2 m-1) \kappa_{2}, & \text { if } \kappa_{1}=0, \kappa_{2}>0 .\end{cases}
$$

Theorem 1.2 is sharp when $\kappa_{1}=\kappa_{2}=0$. In this case, we have $\mu_{1} \geq \bar{\mu}_{1}=\frac{\pi^{2}}{D^{2}}$, giving the same lower bound as in Zhong and Yang ZY84 for Riemannian manifolds with nonnegative Ricci curvature. The sharpness can be seen by constructing a sequence of Kähler manifolds with nonnegative holomorphic sectional curvature and nonnegative orthogonal sectional curvature, which geometrically collapse to the interval $[-D / 2, D / 2]$. For example, one can take the examples constructed in [AC13, Section 5] with $\kappa=0$ for such Kähler manifolds (also notice that the examples in AC13 are not Kähler if $\kappa \neq 0$ ). We suspect that Theorem 1.2 is sharp for $\kappa_{1}=\kappa_{2} \neq 0$ as well.

Next let's turn to the first Dirichlet eigenvalue $\lambda_{1}$. For convenience, denote by $C_{\kappa, \Lambda}(t)$ the unique solution of the initial value problem

$$
\left\{\begin{array}{l}
\phi^{\prime \prime}+\kappa \phi=0, \\
\phi(0)=1, \phi^{\prime}(0)=-\Lambda,
\end{array}\right.
$$

and define $T_{\kappa, \Lambda}$ for $\kappa, \Lambda \in \mathbb{R}$ by

$$
T_{\kappa, \Lambda}(t):=-\frac{C_{\kappa, \Lambda}^{\prime}(t)}{C_{\kappa, \Lambda}(t)} .
$$

In the Riemannian setting, the following result is well-known.

Theorem 1.3. Let $\left(M^{n}, g\right)$ be a compact Riemannian manifold with smooth boundary $\partial M \neq \emptyset$. Suppose that the Ricci curvature of $M$ is bounded from below by $(n-1) \kappa$ and the mean curvature of $\partial M$ is bounded from below by $(n-1) \Lambda$ for some $\kappa, \Lambda \in \mathbb{R}$. Let $\lambda_{1}$ be the first Dirichlet eigenvalue of the Laplacian on $M$. Then

$$
\lambda_{1} \geq \bar{\lambda}_{1}(n, \kappa, \Lambda, R),
$$


where $\bar{\lambda}_{1}(n, \kappa, \Lambda, R)$ is the first eigenvalue of the one-dimensional eigenvalue problem

$$
\left\{\begin{array}{l}
\varphi^{\prime \prime}-(n-1) T_{\kappa, \Lambda} \varphi^{\prime}=-\lambda \varphi \\
\varphi(0)=0, \varphi^{\prime}(R)=0
\end{array}\right.
$$

Here $R$ denotes the inradius of $M$ defined by

$$
R=\sup \{d(x, \partial M): x \in \partial M\} .
$$

Regarding Theorem 1.3, the special case $\kappa=\Lambda=0$ is due to Li and Yau [LY80] and the general case is obtained by Kause Kas84.

Our second main result is a Kähler counterpart of the above theorem.

Theorem 1.4. Let $\left(M^{m}, g, J\right)$ be a compact Kähler manifold with smooth nonempty boundary $\partial M$. Suppose that the holomorphic sectional curvature is bounded from below by $4 \kappa_{1}$ and orthogonal Ricci curvature is bounded from below by $2(m-1) \kappa_{2}$ for some $\kappa_{1}, \kappa_{2} \in \mathbb{R}$, and the second fundamental form on $\partial M$ is bounded from below by $\Lambda \in \mathbb{R}$. Let $\lambda_{1}$ be the first Dirichlet eigenvalue of the Laplacian on $M$. Then

$$
\lambda_{1} \geq \bar{\lambda}_{1}\left(m, \kappa_{1}, \kappa_{2}, \Lambda, R\right)
$$

where $\bar{\lambda}_{1}\left(m, \kappa_{1}, \kappa_{2}, \Lambda, R\right)$ is the first eigenvalue of the one-dimensional eigenvalue problem

$$
\left\{\begin{array}{l}
\varphi^{\prime \prime}-\left(2(m-1) T_{\kappa_{2}, \Lambda}+T_{4 \kappa_{1}, \Lambda}\right) \varphi^{\prime}=-\lambda \varphi \\
\varphi(0)=0, \varphi^{\prime}(R)=0 .
\end{array}\right.
$$

When the boundary is convex, namely $\Lambda=0$, it is easily seen that we have

$$
\bar{\lambda}_{1}\left(m, \kappa_{1}, \kappa_{2}, 0, R\right)=\bar{\mu}_{1}\left(m, \kappa_{1}, \kappa_{2}, R\right) .
$$

Thus we can obtain the same explicit lower bounds and estimates as in Proposition 1.1 for $\bar{\lambda}_{1}\left(m, \kappa_{1}, \kappa_{2}, 0, R\right)$.

The proof of Theorem 1.4 is similar to that of Theorem 1.3 given in Kas84 and it relies on a comparison theorem for the second derivatives of $d(x, \partial M)$ and Barta's inequality. With the help of a generalized Barta's inequality for the $p$-Laplacian (see [LW20, Theorem 3.1]), the same argument indeed yields such lower bounds for the first Dirichlet eigenvalue of the $p$-Laplacian for all $1<p<\infty$.

The paper is organized as follows. In Section 2, we recall the definitions of holomorphic sectional curvature and orthogonal Ricci curvature for Kähler manifolds. Section 3 is devoted to proving the modulus of continuity estimate for a large class of quasilinear parabolic equations, in terms of dimension, diameter and curvature lower bounds. We prove Theorem 1.2 in Section 4, as large time implication of the modulus of continuity estimates. Explicit lower bounds and estimates in Proposition 1.1 will be proved in Section 5. In Section 6, we prove a comparison theorem for the second derivatives of $d(x, \partial M)$, which will be used to prove Theorem 1.4 in Section 7 . 


\section{Curvatures of KÄHler Manifolds}

In this section, we briefly recall the notions holomorphic sectional curvature and orthogonal Ricci curvature for Kähler manifolds.

Let $\left(M^{m}, g, J\right)$ be a Kähler manifold of complex dimension $m$ (real dimension is $n=2 m)$. We regard $M$ as a $2 n$-dimensional Riemannian manifold with a parallel complex structure $J$. A plane $\sigma \subset T_{p} M$ is said to be holomorphic if it is invariant by the complex structure tensor $J$. The restriction of the sectional curvature to holomorphic planes is called the holomorphic sectional curvature and will be denoted by $H$. In other words, if $\sigma$ is a holomophic plane spanned by $X$ and $J X$, then the holomorphic sectional curvature of $\sigma$ is defined by

$$
H(\sigma):=H(X)=\frac{R(X, J X, X, J X)}{|X|^{4}} .
$$

We say the holomorphic sectional curvature is bounded from below by $\kappa \in \mathbb{R}$ (abbreviated as $H \geq \kappa$ ) if $H(\sigma) \geq \kappa$ for all holomorphic planes $\sigma \subset T_{p} M$ and all $p \in M$. by

The orthogonal Ricci curvature, denoted by $\mathrm{Ric}^{\perp}$, is defined for any $X \in T_{p} M$

$$
\operatorname{Ric}^{\perp}(X, X)=\operatorname{Ric}(X, X)-H(X)|X|^{2} .
$$

We say the orthogonal Ricci curvature is bounded from below by $\kappa \in \mathbb{R}$ (abbreviated as $\left.\operatorname{Ric}^{\perp} \geq \kappa\right)$ if $\operatorname{Ric}^{\perp}(X, X) \geq \kappa|X|^{2}$ for all $X \in T_{p} M$ and all $p \in M$. This new curvature $\mathrm{Ric}^{\perp}$ was recently introduced by $\mathrm{Ni}$ and Zheng [NZ18] in the study of Laplace comparison theorems on Kähler manifolds. We refer the readers to [NZ18] NZ19] NWZ18, for a more detailed account on Ric ${ }^{\perp}$ and recent developments.

\section{Modulus of Continuity Estimates on KÄHLer Manifolds}

In the section, we derive modulus of continuity estimates for a large class of quasilinear parabolic equations on Kähler manifolds, in terms of initial oscillation, elapsed time, and lower bounds of holomorphic sectional curvature and orthogonal Ricci curvature. One should compare it with its Riemannian version derived by Andrews and Clutterbuck in $\mathrm{AC} 13$.

Recall that the modulus of continuity $\omega$ of a continuous function $u$ defined on a metric space $(X, d)$ is defined by

$$
\omega(s):=\sup \left\{\frac{u(y)-u(x)}{2}: d(x, y)=2 s\right\} .
$$

In a series of papers $\mathrm{AC09a}, \mathrm{AC09b}, \mathrm{AC11}, \mathrm{AC13}, \mathrm{And15}$, Andrews and Clutterbuck investigated the question of how the modulus of continuity $\omega$ of $u$ evolves when $u$ is evolving by a parabolic partial differential equation on a bounded domain in the Euclidean space or on a compact Riemannian manifold. They managed to prove that for a large class of quasi-linear parabolic equations (see (3.1) below), the modulus of continuity of a solution is a subsolution of the associated one-dimensional equations. This in particular allows them to proved in $\mathrm{AC09b}$ gradients estimates for the graphical solutions of smoothly anisotropic mean curvature flows depending only the initial oscillation, whereas this has not yet been accomplished using direct 
estimates on the gradient. However, it has turned out that the most important applications of the modulus of continuity estimates are the large time implications. For instance, the modulus of continuity estimate leads directly to exponential decay rate for the oscillation of solutions of the heat equation, thus implying a lower bound on the first nonzero eigenvalue of the Laplacian. This observation was used in $\mathrm{AC13}$ (see also [ZW17] for an elliptic proof) to provide a simple alternative proof of the sharp lower bound for the first eigenvalue of the Laplacian in terms of dimension, diameter and lower bound of Ricci curvature (see Theorem 1.1). More surprisingly, this idea was used in AC11] to derive an optimal lower bound on the difference between the first two Dirichlet eigenvalues for the Laplacian on a convex Euclidean domain, thus proving the long-standing Fundamental Gap Conjecture (see also [Ni13] for an elliptic proof and HWZ20] DSW18] SWW19] for fundamental gap of convex domains in the sphere). In another direction, the modulus of continuity estimate has been extended to viscosity solutions in [Li16] [LW17], and to fully nonlinear parabolic equations in [Li20]. We refer the read to the the survey by Andrews And15, where these ideas were further explained and connections to other problems in geometric analysis are made.

As in [AC13] and And15], we consider the following quasilinear isotropic equation

$$
\frac{\partial u}{\partial t}=Q[u]:=\left[\alpha(|\nabla u|) \frac{\nabla_{i} u \nabla_{j} u}{|\nabla u|^{2}}+\beta(|\nabla u|)\left(\delta_{i j}-\frac{\nabla_{i} u \nabla_{j} u}{|\nabla u|^{2}}\right)\right] .
$$

Here $\alpha$ and $\beta$ are smooth positive functions. Some important examples of (3.1) are the heat equation (with $\alpha=\beta=1$ ) and the $p$-Laplacian heat flows (with $\alpha=(p-1)|\nabla u|^{p-2}$ and $\beta=|\nabla u|^{p-2}$ ) and the graphical mean curvature flow (with $\alpha=1 /\left(1+|\nabla u|^{2}\right)$ and $\left.\beta=1\right)$.

To the operator $Q$ defined in (3.1), the associated one-dimensional operator $\mathcal{L}$ is given by

$$
\mathcal{L} \varphi=\alpha\left(\varphi^{\prime}\right) \varphi^{\prime \prime}-\left(2(m-1) T_{\kappa_{2}}+T_{4 \kappa_{1}}\right) \beta\left(\varphi^{\prime}\right) \varphi^{\prime},
$$

where $T_{\kappa}$ is defined by (1.2) .

The main result of this section is the following modulus of continuity estimates on Kähler manifolds.

Theorem 3.1. Let $\left(M^{m}, g, J\right)$ be a compact Kähler manifold with diameter $D$ whose holomorphic sectional section is bounded from below by $4 \kappa_{1}$ and the orthogonal Ricci curvature is bounded from below by $2(m-1) \kappa_{2}$ for some $\kappa_{1}, \kappa_{2} \in \mathbb{R}$. Let $u: M \times[0, T) \rightarrow \mathbb{R}$ be a solution of (3.1) (with Neumann boundary condition if $M$ has a strictly convex boundary). Then the modulus of continuity $\omega:[0, D / 2] \times[0, T) \rightarrow \mathbb{R}$ of $u$ is a viscosity subsolution of the one-dimensional equation

$$
\omega_{t}=\mathcal{L} \omega,
$$

where $\mathcal{L}$ is defined in (3.2).

Proof of Theorem 3.1. The proof is in the same spirit as in AC13, but differs from the choices of variations due to Kählerity and different assumptions on the 
curvatures. It will be convenient to use the function $c_{\kappa}$ defined by

$$
c_{\kappa}(t)= \begin{cases}\cos \sqrt{\kappa} t & \text { if } \kappa>0, \\ 1 & \text { if } \kappa=0, \\ \cosh \sqrt{-\kappa t} & \text { if } \kappa<0 .\end{cases}
$$

By the definition of viscosity solutions (see [CIL92]), we need to show that for every smooth function $\varphi$ that touches $\omega$ from above at $\left(s_{0}, t_{0}\right) \in(0, D / 2) \times(0, T)$ in the sense that

it holds that

$$
\left\{\begin{array}{l}
\varphi(s, t) \geq \omega(s, t) \text { near }\left(s_{0}, t_{0}\right), \\
\varphi\left(s_{0}, t_{0}\right)=\omega\left(s_{0}, t_{0}\right)
\end{array}\right.
$$

$$
\varphi_{t} \leq \mathcal{L} \varphi,
$$

at the point $\left(s_{0}, t_{0}\right)$. It follows from the definition of $\omega$ that for such a function $\varphi$, we have

$$
u(\gamma(1), t)-u(\gamma(0), t)-2 \varphi\left(\frac{L[\gamma]}{2}, t\right) \leq 0
$$

for any $t \leq t_{0}$ close to $t_{0}$ and any smooth path $\gamma:[a, b] \rightarrow M$ with length close to $2 s_{0}$. Moreover, since $M$ is compact, there exist points $x_{0}$ and $y_{0}$ in $M$ (assume for a moment that $\partial M=\emptyset)$, with $d\left(x_{0}, y_{0}\right)=2 s_{0}$ such that the equality in (3.6) holds for $\gamma_{0}:\left[-s_{0}, s_{0}\right] \rightarrow M$, a length-minimizing unit speed geodesic connecting $x_{0}$ and $y_{0}$. The key idea is to derive useful inequalities from the first and second tests along smooth family of variations of the curve $\gamma_{0}$. For this purpose, we need to recall the first and second variation formulas of arc length. If $\gamma:(r, s) \rightarrow \gamma_{r}(s)$ is a smooth variation of $\gamma_{0}(s)$, then we have

$$
\left.\partial_{r} L\left[\gamma_{r}\right]\right|_{r=0}=\left.g\left(T, \gamma_{r}\right)\right|_{-s_{0}} ^{s_{0}}
$$

and

$$
\left.\partial_{r}^{2} L\left[\gamma_{r}\right]\right|_{r=0}=\int_{-s_{0}}^{s_{0}}\left(\left|\left(\nabla_{s} \gamma_{r}\right)^{\perp}\right|^{2}-R\left(\gamma_{s}, \gamma_{r}, \gamma_{s}, \gamma_{r}\right)\right) d s+\left.g\left(T, \nabla_{r} \gamma_{r}\right)\right|_{-s_{0}} ^{s_{0}},
$$

where $T$ is the unit tangent vector to $\gamma_{0}$.

It will be convenient to work in the (complex) Fermi coordinates along $\gamma_{0}$ chosen as follows. We pick an orthonormal basis $\left\{e_{i}\right\}_{i=1}^{2 m}$ for $T_{x_{0}} M$ with $e_{1}=\gamma_{0}^{\prime}\left(-s_{0}\right)$ and $e_{2}=J \gamma_{0}^{\prime}\left(-s_{0}\right)$, where $J$ is the complex structure. Then parallel transport along $\gamma_{0}$ produces an orthonormal basis $\left\{e_{i}(s)\right\}_{i=1}^{2 m}$ for $T_{\gamma_{0}(s)} M$ with $e_{1}(s)=\gamma_{0}^{\prime}(s)$ for each $s \in\left[-s_{0}, s_{0}\right]$. Since $J$ is parallel and $\gamma_{0}$ is a geodesic, the vector field $J \gamma_{0}^{\prime}(s)$ is parallel and thus we have $e_{2}(s)=J e_{1}(s)$ for each $s \in\left[-s_{0}, s_{0}\right]$.

First derivatives consideration implies that

$$
Q[u]\left(y_{0}, t_{0}\right)-Q[u]\left(x_{0}, t_{0}\right)-2 \varphi_{t} \geq 0,
$$

and

$$
\nabla u\left(x_{0}, t_{0}\right)=-\varphi^{\prime} e_{1}\left(-s_{0}\right), \quad \nabla u\left(y_{0}, t_{0}\right)=\varphi^{\prime} e_{1}\left(s_{0}\right) .
$$

Here and below, all derivatives of $\varphi$ are evaluated at $\left(s_{0}, t_{0}\right)$. The variation $\gamma(r, s)=$ $\gamma_{0}\left(s+r \frac{2 s-1}{L}\right)$ has $\gamma_{r}=\frac{2 s-1}{L} \gamma_{0}^{\prime}$, and thus $\partial_{r} L[\gamma]=2$, and $\partial_{r}^{2} L[\gamma]=0$. The second derivative test for this variation produces

$$
u_{11}\left(y_{0}, t_{0}\right)-u_{11}\left(x_{0}, t_{0}\right)-2 \varphi^{\prime \prime} \leq 0 .
$$


Here the subscripts denote covariant derivatives in directions corresponding to the orthonormal basis $\left\{e_{i}\right\}$. Next, we consider the variation $\gamma(r, s)=\exp _{\gamma_{0}(s)}\left(r \eta(s) e_{2}(s)\right)$ for some smooth function $\eta$ to be determined. One easily computes that $\gamma_{r}=$ $\eta(s) e_{2}(s), \partial_{r} L[\gamma]=0$, and

$$
\partial_{r}^{2} L[\gamma]=\int_{-s_{0}}^{s_{0}}\left(\left(\eta^{\prime}\right)^{2}-\eta^{2} R\left(e_{1}, e_{2}, e_{1}, e_{2}\right)\right) d s .
$$

So this variation gives that

$$
u_{22}\left(y_{0}, t_{0}\right)-u_{22}\left(x_{0}, t_{0}\right)-\varphi^{\prime} \int_{-s_{0}}^{s_{0}}\left(\left(\eta^{\prime}\right)^{2}-\eta^{2} R\left(e_{1}, e_{2}, e_{1}, e_{2}\right)\right) d s \leq 0 .
$$

Similarly, for $3 \leq i \leq 2 m$, the variation $\gamma(r, s)=\exp _{\gamma_{0}(s)}\left(r \zeta(s) e_{i}(s)\right)$ with $\zeta$ to be decided, produces

$$
u_{i i}\left(y_{0}, t_{0}\right)-u_{i i}\left(x_{0}, t_{0}\right)-\varphi^{\prime} \int_{-s_{0}}^{s_{0}}\left(\left(\zeta^{\prime}\right)^{2}-\zeta^{2} R\left(e_{1}, e_{i}, e_{1}, e_{i}\right)\right) d s \leq 0 .
$$

Combining (3.9), (3.10), (3.11) together and in view of (3.8), we obtain that

$$
\begin{aligned}
& Q[u]\left(y_{0}, t_{0}\right)-Q[u]\left(x_{0}, t_{0}\right) \\
= & \alpha\left(\varphi^{\prime}\right)\left(u_{11}\left(y_{0}, t_{0}\right)-u_{11}\left(x_{0}, t_{0}\right)\right)+\beta\left(\varphi^{\prime}\right) \sum_{i=2}^{2 m}\left(u_{i i}\left(y_{0}, t_{0}\right)-u_{i i}\left(x_{0}, t_{0}\right)\right) \\
\leq & 2 \alpha\left(\varphi^{\prime}\right) \varphi^{\prime \prime}+\beta\left(\varphi^{\prime}\right) \varphi^{\prime} \int_{-s_{0}}^{s_{0}}\left(\left(\eta^{\prime}\right)^{2}-\eta^{2} R\left(e_{1}, e_{2}, e_{1}, e_{2}\right)\right) d s \\
& +\beta\left(\varphi^{\prime}\right) \varphi^{\prime} \sum_{i=3}^{2 m} \int_{-s_{0}}^{s_{0}}\left(\left(\zeta^{\prime}\right)^{2}-\zeta^{2} R\left(e_{1}, e_{i}, e_{1}, e_{i}\right)\right) d s
\end{aligned}
$$

Choosing $\eta(s)=\frac{c_{4 \kappa}(s)}{c_{4 \kappa}\left(s_{0}\right)}$ yields that

$$
\begin{aligned}
& \int_{-s_{0}}^{s_{0}}\left(\left(\eta^{\prime}\right)^{2}-\eta^{2} R\left(e_{1}, e_{2}, e_{1}, e_{2}\right)\right) d s \\
= & \left.\eta \eta^{\prime}\right|_{-s_{0}} ^{s_{0}}-\int_{-s_{0}}^{s_{0}} \eta^{2}\left(R\left(e_{1}, J e_{1}, e_{1}, J e_{1}\right)-4 \kappa\right) d s \\
\leq & -2 T_{4 \kappa}\left(s_{0}\right),
\end{aligned}
$$

where we have used $\eta^{\prime \prime}+4 \kappa \eta=0$ and the assumption $H \geq 4 \kappa$. Similarly, we obtain with $\zeta(s)=\frac{c_{\kappa}(s)}{c_{\kappa}\left(s_{0}\right)}$ that

$$
\begin{aligned}
& \sum_{i=3}^{2 m} \int_{-s_{0}}^{s_{0}}\left(\left(\zeta^{\prime}\right)^{2}-\zeta^{2} R\left(e_{1}, e_{i}, e_{1}, e_{i}\right)\right) d s \\
= & \int_{-s_{0}}^{s_{0}}\left(2(m-1)\left(\zeta^{\prime}\right)^{2}-\zeta^{2} \operatorname{Ric}^{\perp}\left(e_{1}, e_{1}\right)\right) d s \\
= & \left.2(m-1) \zeta \zeta^{\prime}\right|_{-s_{0}} ^{s_{0}}+\int_{-s_{0}}^{s_{0}} \zeta^{2}\left(\operatorname{Ric}^{\perp}\left(e_{1}, e_{1}\right)-2(m-1) \kappa\right) d s \\
\leq & -2(m-1) T_{\kappa}\left(s_{0}\right),
\end{aligned}
$$

where we have used $\zeta^{\prime \prime}+\kappa \zeta=0$ and $\operatorname{Ric}^{\perp} \geq 2(m-1) \kappa$. Inserting the above two estimates (3.13) and (3.14) into (3.12) gives

$$
Q[u]\left(y_{0}, t_{0}\right)-Q[u]\left(x_{0}, t_{0}\right) \leq 2 \mathcal{L} \varphi .
$$


The desire inequality (3.5) follows immediately by combining (3.7) and (3.15), thus completing the proof if $\partial M=\emptyset$.

Finally, let's deal with the situation that $M$ has a strictly convex boundary. Let $x_{0}$ and $y_{0}$ be such that the function

$$
Z(x, y)=u(y)-u(x)-2 \varphi\left(\frac{d(x, y)}{2}\right)
$$

attains its maximum zero at $\left(x_{0}, y_{0}\right)$. We will rule out the possibility that either $x_{0} \in \partial M$ or $y_{0} \in \partial M$. Without loss of generality, we may assume $x_{0} \in \partial M$. Since $\partial M$ is convex, there exists (see BGS02) a length-minimizing geodesic $\gamma$ : $\left[-s_{0}, s_{0}\right] \rightarrow M$ from $x_{0}$ to $y_{0}$ such that $\gamma(s)$ lies in the interior of $M$ for all $s \in$ $\left(-s_{0}, s_{0}\right)$ and $g\left(\gamma^{\prime}\left(-s_{0}\right), \nu\left(x_{0}\right)\right)>0$, with $\nu\left(x_{0}\right)$ being the inward-pointing unit normal to $\partial M$ at $x_{0}$. Then for $x(s)=\exp _{x_{0}}\left(s \nu\left(x_{0}\right)\right)$, we have

$$
\begin{aligned}
\frac{d}{d s} Z\left(x(s), y_{0}\right) & =-g\left(\nabla u, \nu\left(x_{0}\right)\right)-\varphi^{\prime}\left(s_{0}\right) g\left(-\gamma^{\prime}\left(-s_{0}\right), \nu\left(x_{0}\right)\right) \\
& =\varphi^{\prime}\left(s_{0}\right) g\left(\gamma^{\prime}\left(-s_{0}\right), \nu\left(x_{0}\right)\right)>0 .
\end{aligned}
$$

This contradicts the fact that the function $Z(x, y)$ attains its maximum zero over $M \times M$ at $\left(x_{0}, y_{0}\right)$. The proof is thus complete.

Remark 3.2. Theorem 3.1 remains valid if $u$ is only assumed to be a viscosity solution of (3.1). This will not be needed in the present paper and we refer the interested reader to [Li16] and [WW17] for how to prove modulus of continuity estimates for viscosity solutions.

Remark 3.3. One may follow the approach in [Li20] to derive modulus of continuity estimates for fully nonlinear parabolic equations on Kähler manifolds.

\section{Lower Bounds for the First Nonzero Eigenvalue}

As in the Riemannian setting in [AC13, the large time behavior of the modulus of continuity estimates implies lower bound for the first nonzero eigenvalue of the Laplacian. The goal of this section is to prove lower bounds for the first nonzero eigenvalue of the Laplacian on a Kähler manifold stated in Theorem 1.2, as an application of the modulus of continuity derived in the previous section.

Let's explain the approach of Andrews and Clutterbuck AC13 in more details. The idea to detect the first nonzero eigenvalue (with either $\partial M=\emptyset$ or Neumann boundary condition) of the Laplacian is by knowing how quickly the solutions to the heat equation decay. This is because we may solve

$$
\left\{\begin{array}{l}
u_{t}=\Delta u, \\
u(x, 0)=u_{0}(x),
\end{array}\right.
$$

by expanding $u_{0}=\sum_{i=0}^{\infty} a_{i} \varphi_{i}$, where $\varphi_{i}$ are eigenfunctions of the Laplacian (with Neumann boundary condition if $\partial M \neq \emptyset$ ). Then the solution to the heat equation is given by

$$
u(x, t)=\sum_{i=0}^{\infty} e^{-\mu_{i} t} a_{i} \varphi_{i} .
$$


This does not converges to zero as $\mu_{0}=0$, but the key observation is that $\mid u(x, t)-$ $u(y, t) \mid$ does converges to zero, and in fact

$$
|u(x, t)-u(y, t)| \approx e^{-\mu_{1} t} \text { as } t \rightarrow \infty .
$$

Thus the main step is to establish the estimate

$$
|u(x, t)-u(y, t)| \approx C e^{-\bar{\mu}_{1} t}
$$

for any solution to the heat equation. This turns out to be an easy consequence of the modulus of continuity estimates. Then taking $u(x, t)=e^{-\mu_{1} t} \varphi_{1}(x)$ leads to

$$
\left|\varphi_{1}(x)-\varphi_{1}(y)\right| \leq C e^{\left(\mu_{1}-\bar{\mu}_{1}\right) t},
$$

which implies $\mu_{1} \geq \bar{\mu}_{1}$ by letting $t \rightarrow \infty$.

We begin with an immediate consequence of Theorem 3.1, which asserts that if $\varphi(\cdot, 0)$ lies above the modulus of continuity of $u(\cdot, 0)$ and we evolve $\varphi$ by (3.3), then $\varphi(\cdot, t)$ lies above the modulus of continuity of $u(\cdot, t)$ for each positive $t$.

Corollary 4.1. Let $M$ and $u$ be the same as in Theorem 3.1. Suppose $\varphi:[0, D / 2] \times$ $[0, T) \rightarrow \mathbb{R}$ satisfies

(1) $\varphi_{t} \geq \mathcal{L} \varphi$

(2) $\varphi^{\prime} \geq 0$ on $[0, D / 2] \times[0, T)$;

(3) $|u(y, 0)-u(x, 0)| \leq 2 \varphi\left(\frac{d(x, y)}{2}, 0\right)$.

Then

$$
|u(y, t)-u(x, t)| \leq 2 \varphi\left(\frac{d(x, y)}{2}, t\right)
$$

for all $x, y \in M$ and $t \in[0, T)$.

Proof of Corollary 4.1. For any $\epsilon>0$, the function $\varphi_{\epsilon}=\varphi+\epsilon e^{t}$ satisfies

$$
\left(\varphi_{\epsilon}\right)_{t}>\mathcal{L} \varphi_{\epsilon}
$$

so it cannot touch $\omega$ from above by Theorem 3.1 .

On the interval $[0, D / 2]$, we define the following corresponding one-dimensional eigenvalue problem with boundary conditions $\phi(0)=0$ and $\phi^{\prime}(D / 2)=0$ :

$$
\bar{\sigma}_{1}\left(m, \kappa_{1}, \kappa_{2}, D / 2\right)=\inf \left\{\frac{\int_{0}^{\frac{D}{2}}\left|\phi^{\prime}\right|^{2} c_{\kappa_{2}}^{2 m-2} c_{4 \kappa_{1}} d s}{\int_{0}^{\frac{D}{2}}|\phi|^{2} c_{\kappa_{2}}^{2 m-2} c_{4 \kappa_{1}} d s}, \quad \text { with } \quad \phi(0)=0\right\},
$$

where $c_{\kappa}$ is defined in (3.4).

\section{Lemma 4.1.}

$$
\bar{\mu}_{1}\left(m, \kappa_{1}, \kappa_{2}, D\right)=\bar{\sigma}_{1}\left(m, \kappa_{1}, \kappa_{2}, D / 2\right) .
$$

Proof. Let $\phi(s)$ be an eigenfunction on $[0, D / 2]$ corresponding to $\bar{\sigma}_{1}\left(m, \kappa_{1}, \kappa_{2}, D / 2\right)$. Then for $s \in[-D / 2,0)$ we define $\phi(s)$ by $\phi(s)=-\phi(-s)$. Clearly, $\phi(s)$ defined on $[-D / 2, D / 2]$ is a trial function for $\bar{\mu}_{1}\left(m, \kappa_{1}, \kappa_{2}, D\right)$. Therefore

$$
\bar{\mu}_{1}\left(m, \kappa_{1}, \kappa_{2}, D\right) \leq \bar{\sigma}_{1}\left(m, \kappa_{1}, \kappa_{2}, D / 2\right) .
$$

For the other direction, let $\psi(s)$ be an eigenfunction corresponding to $\bar{\mu}_{1}\left(m, \kappa_{1}, \kappa_{2}, D\right)$. Without of loss of generality we assume further that $\psi\left(s_{0}\right)=0$ for some $s_{0} \in$ 
$[0, D / 2]$. If $s_{0}>0$, we define $\psi(s)=0$ for $s \in\left[0, s_{0}\right)$. Then $\psi(s)$ is a trial function for $\bar{\sigma}_{1}\left(m, \kappa_{1}, \kappa_{2}, D / 2\right)$, and we have

$$
\begin{aligned}
\bar{\sigma}_{1}\left(m, \kappa_{1}, \kappa_{2}, D / 2\right) & \leq \frac{\int_{0}^{\frac{D}{2}}\left|\psi^{\prime}\right|^{2} c_{\kappa_{2}}^{2 m-2} c_{4 \kappa_{1}} d s}{\int_{0}^{\frac{D}{2}}|\psi|^{2} c_{\kappa_{2}}^{2 m-2} c_{4 \kappa_{1}} d s} \\
& =\frac{\int_{s_{0}}^{\frac{D}{2}}\left|\psi^{\prime}\right|^{2} c_{\kappa_{2}}^{2 m-2} c_{4 \kappa_{1}} d s}{\int_{s_{0}}^{\frac{D}{2}}|\psi|^{2} c_{\kappa_{2}}^{2 m-2} c_{4 \kappa_{1}} d s} \\
& =\bar{\mu}_{1}\left(m, \kappa_{1}, \kappa_{2}, D\right) .
\end{aligned}
$$

This proves the lemma.

Lemma 4.2. There exists an odd eigenfunction $\phi$ corresponding to $\bar{\mu}_{1}\left(m, \kappa_{1}, \kappa_{2}, D\right)$ satisfying

$$
\phi^{\prime \prime}-\left(T_{4 \kappa_{1}}+2(m-1) T_{\kappa_{2}}\right) \phi^{\prime}+\bar{\mu}_{1}\left(m, \kappa_{1}, \kappa_{2}, D\right) \phi=0
$$

in $(0, D / 2)$ with $\phi^{\prime}(s)>0$ in $(0, D / 2)$ and $\phi^{\prime}(D / 2)=0$.

Proof. Lemma 4.1 gives the existence of odd eigenfunction $\phi$ corresponding to $\bar{\mu}_{1}\left(m, \kappa_{1}, \kappa_{2}, D\right)$ with boundary conditions $\phi(0)=0$ and $\phi^{\prime}(D / 2)=0$, which does not change sign in $(0, D / 2]$. Since

$$
\left(c_{\kappa_{2}}^{2 m-2} c_{4 \kappa_{1}} \phi^{\prime}\right)^{\prime}=-\bar{\mu}_{1}\left(m, \kappa_{1}, \kappa_{2}, D\right) c_{\kappa_{2}}^{2 m-2} c_{4 \kappa_{1}} \phi,
$$

we can choose $\phi(s)$ such that $\phi^{\prime}(s)>0$ in $(0, D / 2)$.

We provide the proof of Theorem 1.2 below for the sake of completeness.

Proof of Theorem 1.2. For any $D_{1}>D$, let $\bar{\mu}_{1}=\bar{\mu}_{1}\left(m, \kappa_{1}, \kappa_{2}, D_{1}\right)$ be the first nonzero Neumann eigenvalue of the operator $\mathcal{L}$ defined in (3.2) on the interval $\left[-D_{1} / 2, D_{1} / 2\right]$. By Lemma 4.2, the associated eigenfunction $\phi(s)$ is odd and can be chosen so that $\phi>0$ on $\left(0, D_{1} / 2\right]$ and $\phi^{\prime}(0)>0$. Let $u(x)$ be an eigenfunction corresponding to $\mu_{1}$. Then there exists $C>0$ such that

$$
u(y)-u(x)-2 C \phi\left(\frac{d(x, y)}{2}\right) \leq 0
$$

for all $x, y \in M$. Direct calculation shows that the functions $v(x, t):=e^{-\mu_{1} t} u(x)$ and $\varphi(s, t):=C e^{-\bar{\mu}_{1} t} \phi$ satisfy

and

$$
\frac{\partial v}{\partial t}=\Delta v
$$

$$
\frac{\partial \varphi}{\partial t}=\mathcal{L} \varphi
$$

respectively. Moreover, it's easy to verify that $C \varphi$ satisfies all the conditions in Corollary 4.1, and therefore, we have

$$
u(y, t)-u(x, t) \leq 2 C \varphi\left(\frac{d(x, y)}{2}, t\right),
$$

or equivalently,

$$
e^{-\mu_{1} t}(u(y)-u(x)) \leq 2 C e^{-\bar{\mu}_{1} t} \phi\left(\frac{d(x, y)}{2}\right)
$$


for all $x, y \in M$ and $t>0$. Thus as $t \rightarrow \infty$, inequality (4.3) implies

$$
\mu_{1} \geq \bar{\mu}_{1} .
$$

Theorem 1.2 then follows easily because $\bar{\mu}_{1} \rightarrow \bar{\mu}_{1}\left(m, \kappa_{1}, \kappa_{2}, D\right)$ as $D_{1} \rightarrow D$.

Remark 4.2. The modulus of continuity approach can be adapted to prove similar lower bounds for the first nonzero eigenvalue the p-Laplacian for Riemannian manifolds when $1<p \leq 2$. This is outlined in [And15, Section 8] (see also [LW19a, Section 2]). It's not hard to adapt our argument to prove lower bounds for the first nonzero eigenvalue of the p-Laplacian on Kähler manifolds when $1<p \leq 2$. The $p>2$ case can be handled with gradient estimates method (see NV14 Val12 LW19b]) for Riemannian manifolds, but remains open for Kähler manifolds.

\section{Some Explicit Lower Bounds}

In this section, we prove the explicit lower bounds and estimates claimed in Proposition 1.1. Then we compare our lower bound in Theorem 1.2 with Lichnerowicz's lower bound.

Proof of Proposition 1.1. For the $\kappa_{1}=\kappa_{2}=0$ case, simply observe that the corresponding first eigenfunction is given by $\sin \left(\frac{\pi t}{D}\right)$.

If $\kappa_{1}>0$ and $\kappa_{2}=0$, then by a result of Tsukamoto [Tsu57, the diameter is bounded from above by $\frac{\pi}{2 \sqrt{\kappa_{1}}}$, which yield the inequality by domain monotonicity of the first Neumann eigenvalue. The inequality follows by observing that the first eigenfunction on the interval $\left[-\frac{\pi}{4 \sqrt{\kappa_{1}}}, \frac{\pi}{4 \sqrt{\kappa_{1}}}\right]$ is given by $\sin \left(2 \sqrt{\kappa_{1}} t\right)$.

Similarly, if $\kappa_{1}=0$ and $\kappa_{2}>0$, then $\operatorname{Ric}^{\perp} \geq 2(m-1) \kappa_{2}$ implies $D \leq \frac{\pi}{\sqrt{\kappa_{2}}}$

by [NZ18, Theorem 3.2] and the first eigenfunction on $\left[-\frac{\pi}{2 \sqrt{\kappa_{2}}}, \frac{\pi}{2 \sqrt{\kappa_{2}}}\right]$ is $\sin \left(\sqrt{k_{2}} t\right)$ with first nonzero eigenvalue equal to $(2 m-1) \kappa_{2}$.

It's interesting to compare Theorem 1.2 with Lichnerowicz's lower bound in the positive curvature case. For exmaple, if $H \geq 4 \kappa_{1}>0$ and $\operatorname{Ric}^{\perp} \geq 0$, then Proposition 1.1 gives

$$
\mu_{1}\left(m, \kappa_{1}, 0, D\right) \geq \bar{\mu}_{1}\left(m, \kappa_{1}, 0, \frac{\pi}{2 \sqrt{\kappa_{1}}}\right)=8 \kappa_{1},
$$

while Lichnerowicz's lower bound for Kähler manifolds yields $\mu_{1} \geq 8 \kappa_{1}$ since Ric $\geq$ $4 \kappa_{1}>0$. Therefore, we have better (strictly better for any $D$ smaller than the maximal diameter $\frac{\pi}{2 \sqrt{\kappa_{1}}}$ allowed under the condition $H \geq 4 \kappa_{1}>0$ by [Tsu57]) lower bounds under stronger curvature assumptions.

\section{A Comparison Theorem for $d(x, \partial M)$}

Let $\left(M^{m}, g, J\right)$ be a compact Kähler manifold with smooth nonempty boundary $\partial M$. Denote by $d(x, \partial M)$ the distance function to the boundary of $M$ given by

$$
d(x, \partial M)=\inf \{d(x, y): y \in \partial M\} .
$$


Recall the inradius $R$ is given by

$$
R=\sup \{d(x, \partial M): x \in \partial M\} .
$$

In this section, we prove the following comparison theorem for the second derivatives of $d(x, \partial M)$ on a Kähler manifold with boundary.

Theorem 6.1. Let $\left(M^{m}, g, J\right)$ be a compact Kähler manifold with smooth nonempty boundary $\partial M$. Suppose that $H \geq 4 \kappa_{1}$ and $\operatorname{Ric}^{\perp} \geq 2(m-1) \kappa_{2}$ for some $\kappa_{1}, \kappa_{2} \in \mathbb{R}$, and the second fundamental form on $\partial M$ is bounded from below by $\Lambda \in \mathbb{R}$. Let $\varphi:[0, R] \rightarrow \mathbb{R}_{+}$be a smooth function with $\varphi^{\prime} \geq 0$. Then the function $v(x)=$ $\varphi(d(x, \partial M))$ is a viscosity supersolution of

$$
Q[v]=\left.\left[\alpha\left(\varphi^{\prime}\right) \varphi^{\prime \prime}-\beta\left(\varphi^{\prime}\right) \varphi^{\prime}\left(2(m-1) T_{\kappa_{2}, \Lambda}+T_{4 \kappa_{1}, \Lambda}\right)\right]\right|_{d(x, \partial M)},
$$

on $M$, where $Q$ is the operator defined in (3.1).

Proof of Theorem 6.1. By approximation, it suffices to consider the case $\varphi^{\prime}>0$ on $[0, R]$. By definition of viscosity solutions (see [CIL92]), it suffices to prove that for any smooth function $\psi$ touching $v$ from below at $x_{0} \in M$, i.e.,

$$
\psi(x) \leq v(x) \text { on } M, \quad \psi\left(x_{0}\right)=v\left(x_{0}\right),
$$

it holds that

$$
Q[\psi]\left(x_{0}\right) \leq\left.\left[\alpha\left(\varphi^{\prime}\right) \varphi^{\prime \prime}-\beta\left(\varphi^{\prime}\right) \varphi^{\prime}\left(2(m-1) T_{\kappa_{2}, \Lambda}+T_{4 \kappa_{1}, \Lambda}\right)\right]\right|_{d\left(x_{0}, \partial M\right)} .
$$

Since the function $d(x, \partial M)$ may not be smooth at $x_{0}$, so we need to replace it by a smooth function $\bar{d}(x)$ defined in a neighborhood $U\left(x_{0}\right)$ of $x_{0}$ satisfying $\bar{d}(x) \geq$ $d(x, \partial M)$ for $x \in U\left(x_{0}\right)$ and $\bar{d}\left(x_{0}\right)=d\left(x_{0}, \partial M\right)$. The construction is standard (see e.g. Wu79, pp. 73-74] or [AX19, pp. 1187]), which we state below for reader's convenience.

Since $M$ is compact, there exists $y_{0} \in \partial M$ such that

$$
d\left(x_{0}, y_{0}\right)=d\left(x_{0}, \partial M\right):=s_{0} .
$$

Let $\gamma:\left[0, s_{0}\right] \rightarrow M$ be the unit speed length-minimizing geodesic with $\gamma(0)=x_{0}$ and $\gamma\left(s_{0}\right)=y_{0}$. For any vector $X \in \exp _{x_{0}}^{-1} U\left(x_{0}\right)$, let $X(s), s \in\left[0, s_{0}\right]$ be the vector field obtained by parallel translating $X$ along $\gamma$, and decompose it as

$$
X(s)=a X^{\perp}(s)+b \gamma^{\prime}(s)+c J \gamma^{\prime}(s),
$$

where $a, b$ and $c$ are constants along $\gamma$ with $a^{2}+b^{2}+c^{2}=|X|^{2}$, and $X^{\perp}(s)$ is a unit parallel vector field along $\gamma$ orthogonal to $\gamma^{\prime}(s)$ and $J \gamma^{\prime}(s)$. Define

$$
W(s)=a \eta(s) X^{\perp}(s)+b\left(1-\frac{s}{s_{0}}\right) \gamma^{\prime}(s)+c \zeta(s) J \gamma^{\prime}(s),
$$

where $\eta, \zeta:\left[0, s_{0}\right] \rightarrow \mathbb{R}_{+}$are two $C^{2}$ functions to be chosen later. Next we define the $n$-parameter family of curves $\gamma_{X}:\left[0, s_{0}\right] \rightarrow M$ such that

(1) $\gamma_{0}=\gamma$;

(2) $\gamma_{X}(0)=\exp _{x_{0}}(W(0))$ and $\gamma_{X}\left(s_{0}\right) \in \partial M$;

(3) $W(s)$ is induced by the one-parameter family of curves $l \rightarrow \gamma_{l X}(s)$ for $l \in\left[-l_{0}, l_{0}\right]$ and $s \in\left[0, s_{0}\right]$;

(4) $\gamma_{X}$ depends smoothly on $X$. 
Finally let $\bar{d}(x)$ be the length of the curve $\gamma_{X}(x)$ where $x=\exp _{x_{0}}(X) \in U\left(x_{0}\right)$. Then we have $\bar{d}(x) \geq d(x, \partial M)$ on $U\left(x_{0}\right), \bar{d}\left(x_{0}\right)=d\left(x_{0}, \partial M\right)$.

Recall the first and second variation formulas:

$$
\nabla \bar{d}\left(x_{0}\right)=-\gamma^{\prime}(0)
$$

and

$$
\begin{aligned}
\nabla^{2} \bar{d}(X, X)= & -a^{2} \eta\left(s_{0}\right)^{2} A\left(X^{\perp}\left(s_{0}\right), X^{\perp}\left(s_{0}\right)\right)+c^{2} \zeta\left(s_{0}\right)^{2} A\left(J \gamma^{\prime}, J \gamma^{\prime}\right) \\
& +a^{2} \int_{0}^{s_{0}}\left(\left(\eta^{\prime}\right)^{2}-\eta^{2} R\left(X^{\perp}, \gamma^{\prime}, X^{\perp}, \gamma^{\prime}\right)\right) d s \\
& +c^{2} \int_{0}^{s_{0}}\left(\left(\zeta^{\prime}\right)^{2}-\zeta^{2} R\left(J \gamma^{\prime}, \gamma^{\prime}, J \gamma^{\prime}, \gamma^{\prime}\right)\right) d s
\end{aligned}
$$

where $A$ denotes the second fundamental form of $\partial M$ at $y_{0}$. Then for an orthonormal frame $\left\{e_{i}(s)\right\}_{i=1}^{2 m}$ along $\gamma$ with $e_{1}(s)=\gamma^{\prime}(s)$ and $e_{2}(s)=J \gamma^{\prime}(s)$, we have

$$
\nabla \bar{d}\left(x_{0}\right)=-e_{1}(0),
$$

and

$$
\nabla^{2} \bar{d}\left(e_{1}(0), e_{1}(0)\right)=0 .
$$

For $i=2$, we obtain by choosing $\zeta(s)=C_{4 \kappa_{1}, \Lambda}\left(s_{0}-s\right) / C_{4 \kappa_{1}, \Lambda}\left(s_{0}\right)$ with $C_{4 \kappa_{1}, \Lambda}$ defined in (1.3) that

$$
\begin{aligned}
& \nabla^{2} \bar{d}\left(e_{2}(0), e_{2}(0)\right) \\
= & -\zeta\left(s_{0}\right)^{2} A\left(e_{2}\left(s_{0}\right), e_{2}\left(s_{0}\right)\right)+\int_{0}^{s_{0}}\left(\zeta^{\prime}\right)^{2}-\zeta^{2} R\left(J \gamma^{\prime}, \gamma^{\prime}, J \gamma^{\prime}, \gamma^{\prime}\right) d s \\
\leq & -\frac{A\left(J \gamma^{\prime}\left(s_{0}\right), J \gamma^{\prime}\left(s_{0}\right)\right)}{C_{4 \kappa_{1}, \Lambda}\left(s_{0}\right)^{2}}+\int_{0}^{s_{0}}\left(\zeta^{\prime}\right)^{2}-4 \kappa_{1} \zeta^{2} d s \\
= & -\frac{A\left(J \gamma^{\prime}\left(s_{0}\right), J \gamma^{\prime}\left(s_{0}\right)\right)}{C_{4 \kappa_{1}, \Lambda}\left(s_{0}\right)^{2}}+\frac{\Lambda}{C_{4 \kappa_{1}, \Lambda}\left(s_{0}\right)^{2}}-T_{4 \kappa_{1}, \Lambda}\left(s_{0}\right) \\
\leq & -T_{4 \kappa_{1}, \Lambda}\left(s_{0}\right) .
\end{aligned}
$$

For $3 \leq i \leq 2 m$, we have

$$
\nabla^{2} \bar{d}\left(e_{i}(0), e_{i}(0)\right)=-\eta\left(s_{0}\right)^{2} A\left(e_{i}\left(s_{0}\right), e_{i}\left(s_{0}\right)\right)+\int_{0}^{s_{0}}\left(\eta^{\prime}\right)^{2}-\eta^{2} R\left(e_{i}, \gamma^{\prime}, e_{i}, \gamma^{\prime}\right) d s .
$$

Summing over $3 \leq i \leq 2 m$ and choosing $\eta(s)=C_{\kappa_{2}, \Lambda}\left(s_{0}-s\right) / C_{\kappa_{2}, \Lambda}\left(s_{0}\right)$ with $C_{\kappa_{2}, \Lambda}$ defined in (1.3) gives

$$
\begin{aligned}
& \sum_{i=3}^{2 m} \nabla^{2} \bar{d}\left(e_{i}(0), e_{i}(0)\right) \\
= & -\frac{\sum_{i=3}^{2 m} A\left(e_{i}\left(s_{0}\right), e_{i}\left(s_{0}\right)\right)}{C_{\kappa_{2}, \Lambda}\left(s_{0}\right)^{2}}+\int_{0}^{s_{0}} 2(m-1)\left(\eta^{\prime}\right)^{2}-\eta^{2} \operatorname{Ric}^{\perp}\left(\gamma^{\prime}, \gamma^{\prime}\right) d s \\
\leq & -\frac{\sum_{i=3}^{2 m} A\left(e_{i}\left(s_{0}\right), e_{i}\left(s_{0}\right)\right)}{C_{\kappa_{2}, \Lambda}\left(s_{0}\right)^{2}}+\frac{2(m-1) \Lambda}{C_{\kappa_{2}, \Lambda}\left(s_{0}\right)^{2}}-2(m-1) T_{\kappa_{2}, \Lambda}\left(s_{0}\right) \\
\leq & -2(m-1) T_{\kappa_{2}, \Lambda}\left(s_{0}\right) .
\end{aligned}
$$


Since the function $\psi(x)-\varphi(d(x, \partial M))$ attains its maximum at $x_{0}$ and $\varphi^{\prime}>0$, it follows that the function $\psi(x)-\varphi(\bar{d}(x))$ attains a local maximum at $x_{0}$. The first and second derivative tests yield

$$
\nabla \psi\left(x_{0}\right)=-\varphi^{\prime} e_{1}(0), \quad \psi_{11}\left(x_{0}\right) \leq \varphi^{\prime \prime},
$$

and

$$
\psi_{i i}\left(x_{0}\right) \leq \varphi^{\prime} \nabla^{2} \bar{d}\left(e_{i}(0), e_{i}(0)\right)
$$

for $2 \leq i \leq 2 m$, where we used (6.1) and (6.2). Here and below the derivatives of $\varphi$ are all evaluated at $s_{0}=d\left(x_{0}, \partial M\right)$. Thus we have

$$
\begin{aligned}
Q[\psi]\left(x_{0}\right) & =\alpha\left(\varphi^{\prime}\right) \psi_{11}+\beta\left(\varphi^{\prime}\right) \sum_{i=2}^{2 m} \psi_{i i} \\
& \leq \alpha\left(\varphi^{\prime}\right) \varphi^{\prime \prime}+\beta\left(\varphi^{\prime}\right) \varphi^{\prime}\left(\sum_{i=2}^{2 m} \nabla^{2} \bar{d}\left(e_{i}(0), e_{i}(0)\right)\right) \\
& \leq \alpha\left(\varphi^{\prime}\right) \varphi^{\prime \prime}-\beta\left(\varphi^{\prime}\right) \varphi^{\prime}\left(2(m-1) T_{\kappa_{2}, \Lambda}+T_{4 \kappa_{1}, \Lambda}\right) .
\end{aligned}
$$

The proof is complete.

\section{Lower Bounds for the First Dirichlet Eigenvalue}

Let $\bar{\lambda}_{1}:=\bar{\lambda}_{1}\left(m, \kappa_{1}, \kappa_{2}, \Lambda, R\right)$ denote the first eigenvalue of one-dimensional eigenvalue problem

$$
\left\{\begin{array}{l}
\varphi^{\prime \prime}-\left(2(m-1) T_{\kappa_{2}, \Lambda}+T_{4 \kappa_{1}, \Lambda}\right) \varphi^{\prime}=-\lambda \varphi \\
\varphi(0)=0, \varphi^{\prime}(R)=0 .
\end{array}\right.
$$

where $T_{\kappa, \Lambda}$ is defined in (1.4). It's easy to see that

$$
\bar{\lambda}_{1}=\inf \left\{\frac{\int_{0}^{R}\left|\phi^{\prime}\right|^{2} C_{\kappa_{2}, \Lambda}^{2 m-2} C_{4 \kappa_{1}, \Lambda} d s}{\int_{0}^{R}|\phi|^{2} C_{\kappa_{2}, \Lambda}^{2 m-2} C_{4 \kappa_{1}, \Lambda} d s}, \quad \text { with } \quad \phi(0)=0\right\},
$$

where $C_{\kappa, \Lambda}$ is defined in (1.3).

Proof of Theorem 1.4. Let $\varphi$ be an eigenfunction with respect to $\bar{\lambda}_{1}$, then

$$
\varphi^{\prime \prime}-\left(2(m-1) T_{\kappa_{2}, \Lambda}+T_{4 \kappa_{1}, \Lambda}\right) \varphi^{\prime}=-\bar{\lambda}_{1} \varphi
$$

with $\varphi(0)=0$ and $\varphi^{\prime}(R)=0$. Moreover $\varphi$ can be chosen so that $\varphi>0$ on $(0, R]$ and $\varphi^{\prime}>0$ on $[0, R)$, see Lemma 4.2. Define a testing function

$$
v(x):=\varphi(d(x, \partial M)) .
$$

Then using Theorem 6.1 (choosing $\alpha=\beta=1$ ), we have

$$
\Delta v(x) \leq-\bar{\lambda}_{1} v(x)
$$

away from the cut locus of $\partial M$, and thus globally in the distributional sense. Notice that $v(x)>0$ in $M$ and $v(x)=0$ on $\partial M$, then we conclude from Barta's inequality (see [Kas84, Lemma 1.1]) that

$$
\lambda_{1} \geq \bar{\lambda}_{1}
$$

The proof of Theorem 1.4 is complete. 


\section{ACKNOWLEDGMENTS}

We would like to thank Professors Ben Andrews, Lei Ni and Richard Schoen for helpful discussions. We are also grateful to Professor Guofang Wei for pointing out the reference [BS19].

\section{REFERENCES}

[AC09a] Ben Andrews and Julie Clutterbuck. Lipschitz bounds for solutions of quasilinear parabolic equations in one space variable. J. Differential Equations, 246(11):4268-4283, 2009.

[AC09b] Ben Andrews and Julie Clutterbuck. Time-interior gradient estimates for quasilinear parabolic equations. Indiana Univ. Math. J., 58(1):351-380, 2009.

[AC11] Ben Andrews and Julie Clutterbuck. Proof of the fundamental gap conjecture. J. Amer. Math. Soc., 24(3):899-916, 2011.

[AC13] Ben Andrews and Julie Clutterbuck. Sharp modulus of continuity for parabolic equations on manifolds and lower bounds for the first eigenvalue. Anal. PDE, 6(5):10131024, 2013.

[And15] Ben Andrews. Moduli of continuity, isoperimetric profiles, and multi-point estimates in geometric heat equations. In Surveys in differential geometry 2014. Regularity and evolution of nonlinear equations, volume 19 of Surv. Differ. Geom., pages 1-47. Int. Press, Somerville, MA, 2015.

[AX19] Ben Andrews and Changwei Xiong. Gradient estimates via two-point functions for elliptic equations on manifolds. Adv. Math., 349:1151-1197, 2019.

[Bal06] Werner Ballmann. Lectures on Kähler manifolds. ESI Lectures in Mathematics and Physics. European Mathematical Society (EMS), Zürich, 2006.

[BGS02] Rossella Bartolo, Anna Germinario, and Miguel Sánchez. Convexity of domains of Riemannian manifolds. Ann. Global Anal. Geom., 21(1):63-83, 2002.

[BQ00] Dominique Bakry and Zhongmin Qian. Some new results on eigenvectors via dimension, diameter, and Ricci curvature. Adv. Math., 155(1):98-153, 2000.

[BS19] Casey Blacker and Shoo Seto. First eigenvalue of the $p$-Laplacian on Kähler manifolds. Proc. Amer. Math. Soc., 147(5):2197-2206, 2019.

[Cha84] Isaac Chavel. Eigenvalues in Riemannian geometry, volume 115 of Pure and Applied Mathematics. Academic Press, Inc., Orlando, FL, 1984. Including a chapter by Burton Randol, With an appendix by Jozef Dodziuk.

[CIL92] Michael G. Crandall, Hitoshi Ishii, and Pierre-Louis Lions. User's guide to viscosity solutions of second order partial differential equations. Bull. Amer. Math. Soc. (N.S.), 27(1):1-67, 1992.

[DSW18] Xianzhe Dai, Shoo Seto, and Guofang Wei. Fundamental gap estimate for convex domains on sphere - the case $n=2$. Comm. Anal. Geom., to appear, arXiv:1803.01115, 2018.

[GKY13] Vincent Guedj, Boris Kolev, and Nader Yeganefar. A Lichnerowicz estimate for the first eigenvalue of convex domains in Kähler manifolds. Anal. PDE, 6(5):1001-1012, 2013.

[HWZ20] Chenxu He, Guofang Wei, and Qi S. Zhang. Fundamental gap of convex domains in the spheres. Amer. J. Math., 142(4):1161-1192, 2020.

[Kas84] Atsushi Kasue. On a lower bound for the first eigenvalue of the Laplace operator on a Riemannian manifold. Ann. Sci. École Norm. Sup. (4), 17(1):31-44, 1984.

[Krö92] Pawel Kröger. On the spectral gap for compact manifolds. J. Differential Geom., 36(2):315-330, 1992.

[Li79] Peter Li. A lower bound for the first eigenvalue of the Laplacian on a compact manifold. Indiana Univ. Math. J., 28(6):1013-1019, 1979.

[Li12] Peter Li. Geometric analysis, volume 134 of Cambridge Studies in Advanced Mathematics. Cambridge University Press, Cambridge, 2012.

[Li16] Xiaolong Li. Moduli of continuity for viscosity solutions. Proc. Amer. Math. Soc., 144(4):1717-1724, 2016. 
[Li20] Xiaolong Li. Modulus of continuity estimates for fully nonlinear parabolic equations. arXiv:2006.16631, 2020. .

[Lic58] André Lichnerowicz. Géométrie des groupes de transformations. Travaux et Recherches Mathématiques, III. Dunod, Paris, 1958.

[LL10] Jun Ling and Zhiqin Lu. Bounds of eigenvalues on Riemannian manifolds. In Trends in partial differential equations, volume 10 of Adv. Lect. Math. (ALM), pages 241-264. Int. Press, Somerville, MA, 2010.

[LW05] Peter Li and Jiaping Wang. Comparison theorem for Kähler manifolds and positivity of spectrum. J. Differential Geom., 69(1):43-74, 2005.

[LW17] Xiaolong Li and Kui Wang. Moduli of continuity for viscosity solutions on manifolds. J. Geom. Anal., 27(1):557-576, 2017.

[LW19a] Xiaolong Li and Kui Wang. Sharp lower bound for the first eigenvalue of the weighted p-Laplacian. arxiv:1910.02295,, 2019.

[LW19b] Xiaolong Li and Kui Wang. Sharp lower bound for the first eigenvalue of the weighted p-Laplacian II. Math. Res. Lett, to appear, arXiv:1911.04596, 2019.

[LW20] Xiaolong Li and Kui Wang. First Robin eigenvalue of the $p$-Laplacian on Riemannian manifolds. Math. Z., to appear, arXiv:2002.06472, 2020.

[LY80] Peter Li and Shing Tung Yau. Estimates of eigenvalues of a compact Riemannian manifold. In Geometry of the Laplace operator (Proc. Sympos. Pure Math., Univ. Hawaii, Honolulu, Hawaii, 1979), Proc. Sympos. Pure Math., XXXVI, pages 205-239. Amer. Math. Soc., Providence, R.I., 1980.

[Ni13] Lei Ni. Estimates on the modulus of expansion for vector fields solving nonlinear equations. J. Math. Pures Appl. (9), 99(1):1-16, 2013.

[NV14] Aaron Naber and Daniele Valtorta. Sharp estimates on the first eigenvalue of the $p$ Laplacian with negative Ricci lower bound. Math. Z., 277(3-4):867-891, 2014.

[NWZ18] Lei Ni, Qingsong Wang, and Fangyang Zheng. Manifolds with positive orthogonal ricci curvature. arXiv:1806.10233v2, 2018.

[NZ18] Lei Ni and Fangyang Zheng. Comparison and vanishing theorems for Kähler manifolds. Calc. Var. Partial Differential Equations, 57(6):Art. 151, 31, 2018.

[NZ19] Lei Ni and Fangyang Zheng. On orthogonal Ricci curvature. In Advances in complex geometry, volume 735 of Contemp. Math., pages 203-215. Amer. Math. Soc., Providence, RI, 2019.

[Rei77] Robert C. Reilly. Applications of the Hessian operator in a Riemannian manifold. Indiana Univ. Math. J., 26(3):459-472, 1977.

[SWW19] Shoo Seto, Lili Wang, and Guofang Wei. Sharp fundamental gap estimate on convex domains of sphere. J. Differential Geom., 112(2):347-389, 2019.

[SY94] R. Schoen and S.-T. Yau. Lectures on differential geometry. Conference Proceedings and Lecture Notes in Geometry and Topology, I. International Press, Cambridge, MA, 1994.

[Tsu57] Yôtarô Tsukamoto. On Kählerian manifolds with positive holomorphic sectional curvature. Proc. Japan Acad., 33:333-335, 1957.

[TY12] Luen-Fai Tam and Chengjie Yu. Some comparison theorems for Kähler manifolds. Manuscripta Math., 137(3-4):483-495, 2012.

[Ura87] Hajime Urakawa. Stability of harmonic maps and eigenvalues of the Laplacian. Trans. Amer. Math. Soc., 301(2):557-589, 1987.

[Val12] Daniele Valtorta. Sharp estimate on the first eigenvalue of the $p$-Laplacian. Nonlinear Anal., 75(13):4974-4994, 2012.

[Wu79] H. Wu. An elementary method in the study of nonnegative curvature. Acta Math., 142(1-2):57-78, 1979.

[ZW17] Yuntao Zhang and Kui Wang. An alternative proof of lower bounds for the first eigenvalue on manifolds. Math. Nachr., 290(16):2708-2713, 2017.

[ZY84] Jia Qing Zhong and Hong Cang Yang. On the estimate of the first eigenvalue of a compact Riemannian manifold. Sci. Sinica Ser. A, 27(12):1265-1273, 1984. 
(to start) Department of Mathematics and Statistics, McMaster University, Hamilton, Ontario, L8S 4K1, Canada

Email address: lxlthu@gmail

School of Mathematical Sciences, Soochow University, Suzhou, 215006, China

Email address: kuiwang@suda.edu.cn 